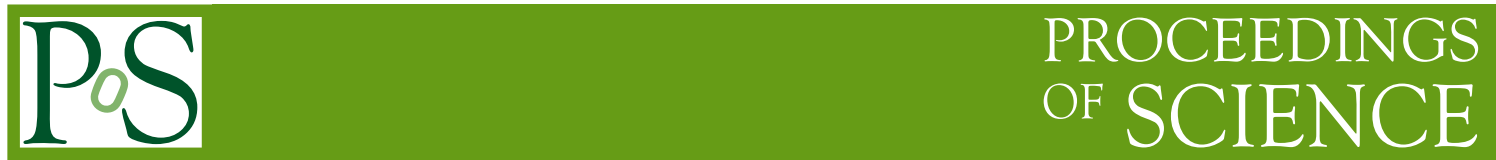

\title{
QCD Phase Transition and the Distribution of Low-lying Eigenvalues with 2+1 Flavors of DWF
}

\section{Zhongjie Lin*}

Department of Physics, Columbia University, New York, NY 10027, USA

E-mail: jasperephys.columbia.edu

Results are reported from seven finite temperature ensembles in the temperature range $T=139-$ $195 \mathrm{MeV}$ with $2+1$ flavors of domain wall fermions and the DSDR gauge action, which all lie on a line of constant physics with a pion mass of $200 \mathrm{MeV}$ and a lattice volume of $32^{3} \times$ 8. We compare preliminary results for the chiral susceptibility and other observables with a previous study at smaller volume and discuss the effects of finite volume. Low-lying eigenvalues of the Dirac operator are computed and related to the breaking of the chiral and anomalous $U(1)_{A}$ symmetries in the region of the QCD phase transition.

The 30th International Symposium on Lattice Field Theory

June 24 - 29, 2012

Cairns, Australia

\footnotetext{
${ }^{*}$ Speaker.
} 


\section{Introduction}

Phase transition of Quantum Chromodynamics (QCD) has remained an intriguing phenomenon for decades in both experimental and theoretical studies. Although it is expected that the dynamically broken $S U(2)_{L} \times S U(2)_{R}$ chiral symmetry will be restored above the critical temperature $T_{c}$, the nature of the phase transition as well as the location of $T_{c}$ is yet to be definitively determined. On the other hand, the $U(1)_{A}$ symmetry is spoiled by the anomaly. Whether it will be effectively restored and whether this restoration coincides with the chiral phase transition remain puzzles to be resolved as well.

Lattice QCD has provided a valuable ab initio method to explore QCD thermodynamics and its associated symmetries. However, studies with common fermionic discretization mechanisms (Wilson, staggered) [ [ [ [] ] suffer from a significant loss of chiral symmetry before the continuum limit is taken. In contrast, domain wall fermions (DWF) [B], 团], which accurately respect the chiral symmetry and reproduce the anomaly, may give a more accurate results at finite lattice spacing.

Previously, we have simulated QCD thermodynamics with DWF on a relatively small $16^{3} \times 8$

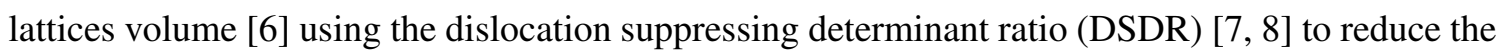
residual chiral symmetry breaking which allowed us to explore a line of constant physics with a $200 \mathrm{MeV}$ pion mass. Here we extend these studies to a larger $32^{3} \times 8$ lattice volume.

We also report the study for the low-lying eigenvalues for the domain wall Dirac operator. The rich physics contained in the eigenvalue density provides a unique perspective on many aspects of the phase transition. The Dirac eigenvalue spectrum can be directly related to both the chiral and $U(1)_{A}$ symmetry breaking and restoration through Banks-Casher type formulae.

\section{Ensembles and Chiral Observables}

Since this is a simple extension of our previous work on $16^{3} \times 8$ lattices [四], we adopt the same gauge and fermion actions and study the same series of seven temperatures in the range of $T=139-195 \mathrm{MeV}$. As in this earlier study, the input light quark masses are adjusted so that all ensembles lie on a line of constant physics with $m_{\pi} \approx 200 \mathrm{MeV}$, with $\widetilde{m}_{l} / \widetilde{m}_{s}=0.088$ to ensure a physical kaon. (Here the tilde indicates the sum of the input and residual quark masses.)

Table $\square$ summarizes the input parameters for our finite temperature ensembles ${ }^{1}$. The use of a

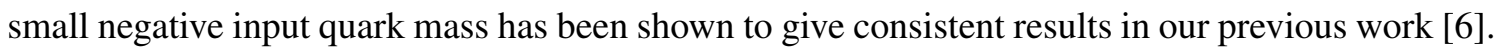
Now with a larger volume, we expect those evolutions with negative input quark masses at $T=139$ $\mathrm{MeV}$ and $149 \mathrm{MeV}$ to be more stable [13]].

The chiral condensates and their susceptibilities are also presented in Table $\mathbb{W}$. In addition to the one-flavor scalar chiral condensates, we introduce a subtracted chiral condensate,

$$
\Delta_{l, s}=\langle\bar{\psi} \psi\rangle_{l}-\frac{m_{l}}{m_{s}}\langle\bar{\psi} \psi\rangle_{s}
$$

in order to remove the divergent piece proportional to the quark mass. We can also compute the disconnected chiral susceptibilities,

$$
\frac{\chi_{l, d i s c}}{T^{2}}=N_{\sigma}^{3} N_{\tau}^{3}\left(\left\langle(\bar{\psi} \psi)_{l}^{2}\right\rangle-\langle\bar{\psi} \psi\rangle_{l}^{2}\right)
$$

\footnotetext{
${ }^{1}$ Additional statistics have been collected since the lattice conference and are included here.
} 


\begin{tabular}{ccc|ccc|cccc|c}
\hline$T(\mathrm{MeV})$ & $\beta$ & $L_{s}$ & $m_{l}$ & $m_{s}$ & $m_{\mathrm{res}}$ & $\langle\bar{\psi} \psi\rangle_{l} / T^{3}$ & $\Delta \bar{\psi} \psi / T^{3}$ & $\chi_{l, d i s c} / T^{2}$ & $\chi_{l, \overline{\mathrm{MS}}} / T^{2}$ & $N_{\text {traj }}$ \\
\hline 139 & 1.633 & 48 & -0.00136 & 0.0519 & $0.00588(39)$ & $10.07(4)$ & $11.16(4)$ & $20(2)$ & $9.5(7)$ & 3000 \\
149 & 1.671 & 32 & -0.00189 & 0.0464 & $0.00643(9)$ & $7.03(6)$ & $8.52(6)$ & $28(3)$ & $12(1)$ & 3000 \\
159 & 1.707 & 32 & 0.000551 & 0.0449 & $0.00377(11)$ & $5.80(6)$ & $5.39(6)$ & $31(3)$ & $14(1)$ & 2943 \\
168 & 1.740 & 32 & 0.00175 & 0.0427 & $0.00209(9)$ & $4.16(8)$ & $2.90(8)$ & $37(3)$ & $16(1)$ & 3008 \\
177 & 1.771 & 32 & 0.00232 & 0.0403 & $0.00132(6)$ & $3.17(5)$ & $1.56(5)$ & $22(2)$ & $9.2(1)$ & 3000 \\
186 & 1.801 & 32 & 0.00258 & 0.0379 & $0.00076(3)$ & $2.46(4)$ & $0.73(4)$ & $12(2)$ & $5.0(8)$ & 3029 \\
195 & 1.829 & 32 & 0.00265 & 0.0357 & $0.00047(1)$ & $2.15(3)$ & $0.43(3)$ & $7(1)$ & $2.8(5)$ & 3412 \\
\hline
\end{tabular}

Table 1: Summary of the parameters and the chiral observables for the finite temperature ensembles with lattice volume of $32^{3} \times 8$. (A minus sign is suppressed for all the chiral condensates). The residual masses are extracted from our $16^{3} \times 8$ ensembles [ [G].

The disconnected piece of the chiral susceptibility, after proper renormalization (e.g. in our case the $\overline{\mathrm{MS}}(\mu=2 \mathrm{GeV})$ scheme), provides a good signature for identifying the crossover temperature as illustrated in Fig. $\mathbf{~}$.
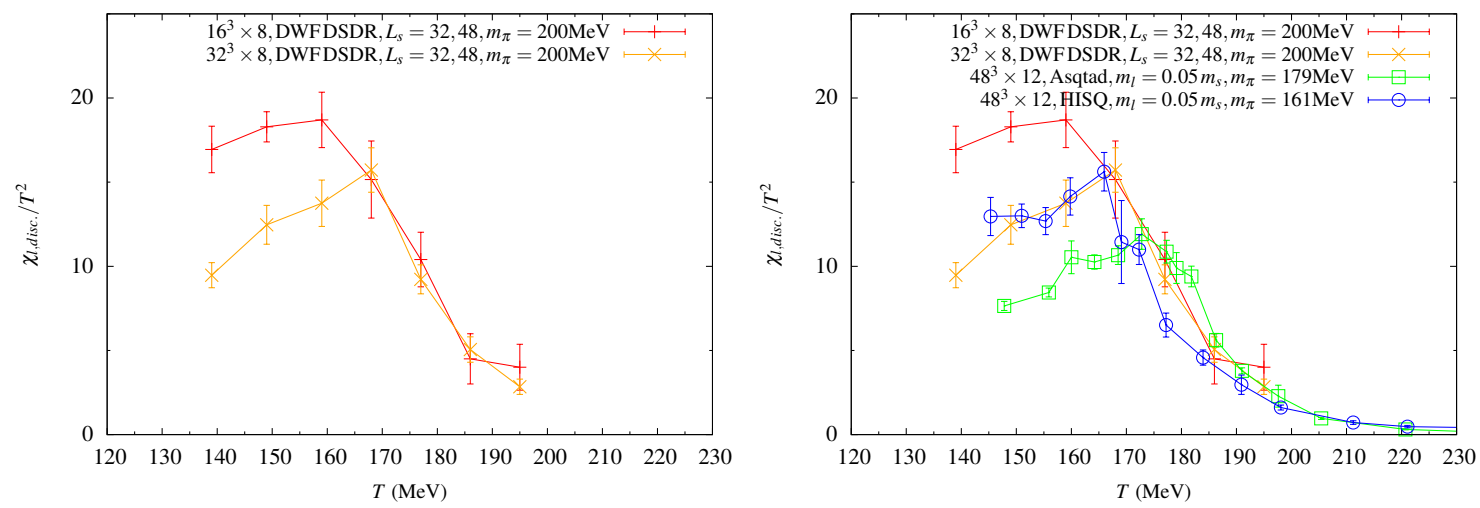

Figure 1: The left panel shows the comparison of the renormalized $\chi_{l, d i s c}$ in our calculation with earlier results [ [ $]$ on a smaller lattice. The right panel shows the comparison of the renormalized $\chi_{l, \text { disc }}$ with results from various staggered actions [四].

The left panel of Fig. $\square$ compares our current calculation with the earlier results from [6] on a smaller lattice. They agree to great extent in regions above the phase transition, indicating the sufficiency of spatial volume size above the QCD phase transition. However, the disconnected susceptibilities from the current $32^{3} \times 8$ work are suppressed significantly below the transition region compared with those from the smaller ensembles. This behavior agrees with the prediction from a scaling analysis of the effects of finite volume using a quark meson model [12]. Despite the fact that a more distinct peak can be observed around $169 \mathrm{MeV}$ for the larger volume, it may be premature to draw a definite conclusion about the pseudo-critical temperature without simulations at a larger volume, a lighter quark mass and another lattice spacing to allow a continuum extrapolation.

The right panel of Fig. $\square$ shows comparison of $\chi_{l, d i s c}^{\overline{\mathrm{MS}}}$ from DWF actions with results from a 
variety of staggered actions [四]. It appears DWF results of volume $32^{3} \times 8$ are quite consistent with $48^{3} \times 12$ staggered results both below and above the transition region, especially with the HISQ action. However, this agreement could be accidental because the results are obtained for different pion masses. There is a factor of 1.25 between the pion mass of the domain wall ensembles and the mass of the lightest Goldstone meson in the HISQ ensembles. One would expect the susceptibilities for DWF would increase appreciably if the pion mass is reduced to the same level as used in the HISQ simulations.

\section{The Low-lying Eigenvalue Distribution of Dirac Operator}

In the exploration of the possible restoration of $U(1)_{A}$ symmetry as well as the chiral symmetry of QCD, the low-lying part of the spectrum of the Dirac operator serves as a unique tool, offering more information than comes from a study of correlators alone. For instance, the order parameter for the $S U(2)_{L} \times S U(2)_{R}$ chiral symmetry and an important $U(1)_{A}$-breaking difference of susceptibilities can be expressed in terms of the eigenvalue density of the Dirac operator [Q, प0],

$$
\begin{aligned}
-\langle\bar{\psi} \psi\rangle_{q} & =\int \mathrm{d} \lambda \rho(\lambda) \frac{2 m_{q}}{m_{q}^{2}+\lambda^{2}}, \quad q=l, s \\
\Delta_{\pi-\delta} & \equiv \chi_{\pi}-\chi_{\delta}=\int \mathrm{d} \lambda \rho(\lambda) \frac{4 m_{l}^{2}}{\left(m_{l}^{2}+\lambda^{2}\right)^{2}} .
\end{aligned}
$$

Different low-lying eigenvalue distributions will lead to different degrees of $U(1)_{A}$ restoration.

To obtain the renormalized eigenvalue spectrum, we follow the same method as described in detail in [6]. The lowest 100 eigenvalues $(\Lambda)$ of the hermitian version of DWF Dirac operator $\left(D_{H} \equiv\right.$ $R_{5} \gamma^{5} D_{\mathrm{DWF}}$ ) with a unitary light quark mass are collected with Kalkreuter-Simma method [W]]. The five-dimensional eigenvalue density is renormalized to a more conventional density in a fashion consistent with the usual bare quark mass $\widetilde{m}$, which enables the removal of the quark mass from the eigenvalue via the relation $|\Lambda|=\sqrt{\lambda^{2}+\widetilde{m}_{l}^{2}}$. The residual mass and therefore the total bare quark mass $\widetilde{m}$ is not well defined on a single configuration at finite lattice spacing. Therefore, when $\widetilde{m}$ is removed from the eigenvalue $\Lambda$, some "unphysical", imaginary values of $\lambda$ appear. We plot these using the negative real number $-\sqrt{\left|\Lambda^{2}-\widetilde{m}_{l}^{2}\right|}$.

Figure $\square$ shows the renormalized distributions of low-lying eigenvalues at various temperatures. The spectra from the $32^{3} \times 8$ ensembles are plotted as histograms, while those from the $16^{3} \times 8$ ensembles [6] are plotted with a black solid line. The distribution from both volumes agree consistently all through the transition region. With larger volume, the same number of modes are more condensed at the lower-end of the spectrum and show less fluctuation, revealing the behavior of the distribution in a more convincing manner. For $T=149-178 \mathrm{MeV}$, the spectra display a linear profile, whose intercept decreases with increasing temperatures and finally vanishes around 168 $\mathrm{MeV}$. This coincides with the peak of the disconnected chiral susceptibility and serves as a good indication of the pseudo-critical temperature for the chiral phase transition via the Banks-Casher relation. For even higher temperatures, a quadratic function instead of a linear one gives a more accurate description of the distribution.

In order to study the anomalous $U(1)_{A}$ symmetry, we attempt to evaluate the difference $\Delta_{\pi-\delta}$ from parameters determined by fitting the eigenvalue spectrum. We assume the eigenvalue density 

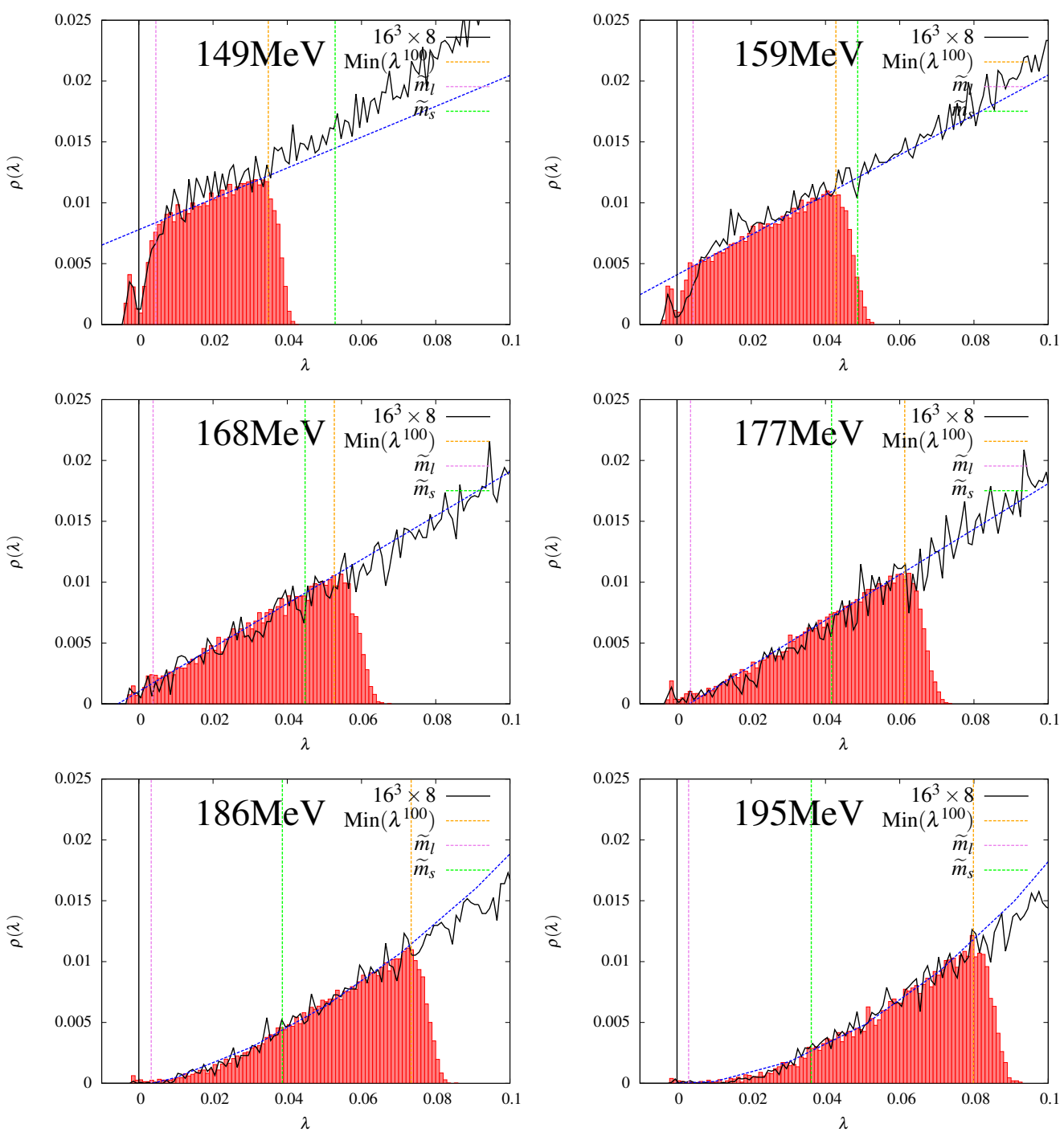

Figure 2: The renormalized eigenvalue spectrum for $T=149-195 \mathrm{MeV}$. The imaginary, "unphysical" eigenvalues are plotted as $-\sqrt{\left|\Lambda^{2}-\widetilde{m}_{l}^{2}\right|}$. The spectra from the $32^{3} \times 8$ ensembles are plotted as histograms and fitted with a linear $(T=149-178 \mathrm{MeV})$ or a quadratic $(T=186-195 \mathrm{MeV})$ function (blue dashed line). The spectra from the $16^{3} \times 8$ ensembles [ $[$ ] are plotted as a black solid line.

$\rho(\lambda)$ has the following expansion near $\lambda=0$ with delta-function, linear and constant terms,

$$
\rho(\lambda)=c_{0} \widetilde{m}^{2} \delta(0)+c_{1} \lambda+c_{2} \widetilde{m}+\cdots
$$

Equation 3.2 then implies that $\Delta_{\pi-\delta}$ will receive the following three contributions:

$$
\frac{\chi_{\pi}-\chi_{\delta}}{T^{2}} \approx 4 c_{0} N_{\tau}^{2}+2 c_{1} N_{\tau}^{2}+\pi c_{2} N_{\tau}^{2} \equiv \chi_{0}^{\pi-\delta}+\chi_{1}^{\pi-\delta}+\chi_{2}^{\pi-\delta} .
$$

Table $\square$ compares these three possible contributions to $\Delta_{\pi-\delta}$ with the corresponding values extracted from correlator measurements on the $16^{3} \times 8$ ensembles. Although the results do not 


\begin{tabular}{cccc|cccc}
\hline$T(\mathrm{MeV})$ & $\beta$ & $\widetilde{m}$ & $Z_{m}$ & $\chi_{0}^{\pi-\delta}$ & $\chi_{1}^{\pi-\delta}$ & $\chi_{2}^{\pi-\delta}$ & $\chi_{\text {corr }}$ \\
\hline 149 & 1.671 & 0.00459 & 1.49 & - & $7.3(5)$ & $174(4)$ & $91(4)$ \\
159 & 1.707 & 0.004321 & 1.51 & - & $9.2(2)$ & $84(2)$ & $78(9)$ \\
168 & 1.740 & 0.00384 & 1.53 & - & $9.8(2)$ & $22(2)$ & $55(10)$ \\
177 & 1.771 & 0.00364 & 1.55 & 47 & $10.0(1)$ & - & $36(14)$ \\
186 & 1.801 & 0.00334 & 1.57 & 16 & - & - & $6(2)$ \\
195 & 1.829 & 0.00302 & 1.58 & 14 & - & - & $8(4)$ \\
\hline
\end{tabular}

Table 2: Comparison of $\Delta_{\pi-\delta}$ from three contributions computed from the fitting of the eigenvalue density on the $32^{3} \times 8$ ensembles to the value extracted from correlator measurements on $16^{3} \times 8$ ensembles $\left(\chi_{\text {corr }}\right)$. Here all results are renormalized to $\overline{\mathrm{MS}}(\mu=2 \mathrm{GeV})$ and the renormalization factors for the quark mass $Z_{m}$ are presented as well.

\begin{tabular}{c|cccccc}
\hline$N_{+}$ & 0 & 1 & 2 & 3 & 4 & 5 \\
\hline$N_{0}=1$ & 28 & 19 & - & - & - & - \\
$N_{0}=2$ & 16 & 19 & 12 & - & - & - \\
$N_{0}=3$ & 4 & 11 & 8 & 3 & - & - \\
$N_{0}=4$ & 1 & 3 & 4 & 3 & 0 & - \\
$N_{0}=5$ & 0 & 2 & 1 & 1 & 1 & 0 \\
\hline
\end{tabular}

Table 3: Number of configurations at $177 \mathrm{MeV}$ carrying each of the possible values for $N_{0}$ and $N_{+}$. Here $N_{0}$ and $N_{+}$are the total number of "near zero modes" and the number of those modes with positive chirality respectively.

agree perfectly due to finite volume effects as well as our crude model, possible trends can be observed. The constant term dominates below the transition region. In contrast, the singular term becomes the major contribution well above the transition.

If this singular contribution results from exact zero modes and non-trivial topology, it should diminish in proportion to $1 / \sqrt{V}$ with increasing space-time volume. If this is the case and the linear term truly vanishes, one might conclude that $U(1)_{A}$ is effective restored. However, if we take a closer look at the eigenvalue distribution near the origin for $T=177-195 \mathrm{MeV}$ (see Fig. [ plotted versus $\Lambda$ in order to preserve the otherwise distorted peak near the origin), rather than shrink by a factor of $\sqrt{8}$, the integrated "near-zero modes" remain approximately the same size when the volume has increased by a factor of 8 . This $\propto V$ behavior is in accordance with the prediction of the dilute instanton gas model, suggesting near zero modes coming from instantons and anti-instantons.
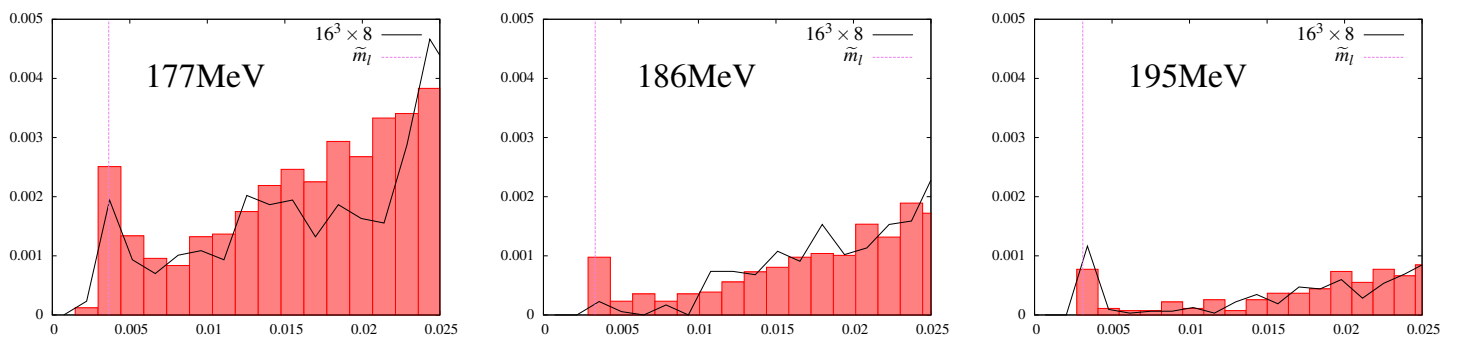

Figure 3: (Left to right) The renormalized eigenvalue spectrum for $T=177-195 \mathrm{MeV}$ without the removal of the bare quark mass. Statistics are insufficient for $186 \mathrm{MeV}$ on $16^{3} \times 8$ ensemble; only 5 instances of "near-zero modes" are collected.

Moreover, if these were exact zero modes forced by non-zero topology, they all should be of the same chirality within one configuration. If they result from a dilute instanton gas, modes of positive and negative chiralities should appear with roughly equal numbers within one configuration. 
From the $177 \mathrm{MeV}$ ensemble, we pick out "near-zero modes" whose chirality is greater than 0.7 in magnitude and in Table 3 list the number of configurations showing each combination of total near zero modes, $N_{0}$, and number of positive chirality modes, $N_{+}$. A binomial distribution consistent with the dilute instanton gas model apparently describes the data better than a bimodal distribution required by exact zero modes, providing additional supporting evidence for the origin of these "near-zero modes". Therefore, $U(1)_{A}$ symmetry probably remains broken at $T=177-195 \mathrm{MeV}$ due to a finite contribution from a dilute instanton gas.

\section{Conclusion}

With chiral symmetry under good control, our $32^{3} \times 8$ simulations with domain wall fermions provide important information about both the chiral and $U(1)_{A}$ symmetries in the vicinity of the QCD phase transition. Chiral condensates and the disconnected susceptibilities are consistent with results from $16^{3} \times 8$ ensembles with a pseudo-critical temperature around $170 \mathrm{MeV}$. Large finite volume effects are observed, especially below the transition.

The eigenvalue spectrum of the DWF Dirac operator is also utilized to study the breaking and restoration of the symmetries that drive the transition. Evidence from a preliminary quantitative analysis of the distribution of the low-lying eigenvalues suggests that $U(1)_{A}$ symmetry is not restored in the region $T=177-195 \mathrm{MeV}$ but is instead explicitly broken in a fashion consistent with the dilute instanton gas model. We are looking forward to a simulation with physical pion masses using Möbius fermions to confirm our findings.

I very much appreciate the help and advice from members of HotQCD and my advisors and colleagues at Columbia University. This work was supported in part by U.S. DOE grant DE-FG0292ER40699. The simulations were carried out on the DOE- and RIKEN-funded QCDOC machines and the NYBlue machine at BNL.

\section{References}

[1] A. Bazavov, et al., Phys.Rev. D85, 054503 (2012), 1111.1710.

[2] S. Borsanyi, et al., JHEP 1009, 073 (2010), 1005.3508.

[3] D. B. Kaplan, Phys.Lett. B288, 342-347 (1992), hep-lat/ 9206013.

[4] V. Furman, and Y. Shamir, Nucl.Phys. B439, 54-78 (1995), hep-lat/ 9405004.

[5] M. Cheng, et al., Phys.Rev. D81, 054510 (2010), 0911.3450.

[6] A. Bazavov, et al., 1205.3535.

[7] P. M. Vranas, hep-lat/0001006.

[8] H. Fukaya, et al., Phys.Rev. D74, 094505 (2006), hep-lat / 0607020.

[9] T. Banks, and A. Casher, Nucl.Phys. B169, 103 (1980),

[10] S. Chandrasekharan and N.H. Christ, Nucl.Phys.Proc.Suppl. 47, 527 (1995), hep-lat/ 9509095.

[11] T. Kalkreuter, and H. Simma, Comput.Phys.Commun. 93, 33-47 (1996), hep-lat/ 9507023.

[12] J. Braun, et al., Eur.Phys.J. C71, 1576 (2011), 1008.2155.

[13] L. Del Debbio, et al., JHEP 0602, 011 (2006), hep-lat/ 0512021. 